\title{
Energy localization in the Peyrard-Bishop DNA model
}

\author{
Jayme De Luca, ${ }^{1, *}$ Elso Drigo Filho, ${ }^{2}$ Antonio Ponno, ${ }^{3}$ and José Roberto Ruggiero ${ }^{2}$ \\ ${ }^{1}$ Universidade Federal de São Carlos, Departamento de Física, Rodovia Washington Luis, km 235, São Carlos, \\ 13565-905-São Paulo, Brazil \\ ${ }^{2}$ Universidade Estadual Paulista “Julio de Mesquita Filho," Instituto de Biociências, letras e Ciências Exatas, Departamento de Física, \\ Rua Cristovão Colombo 2265, São José do Rio Preto, 15054-000-São Paulo, Brazil \\ ${ }^{3}$ Università degli Studi di Milano, Dipartimento di Matematica “F. Enriques," Via Saldini 50, 20133 Milano, Italy
}

(Received 14 April 2004; published 27 August 2004)

\begin{abstract}
We study energy localization on the oscillator chain proposed by Peyrard and Bishop to model DNA. We search numerically for conditions with initial energy in a small subgroup of consecutive oscillators of a finite chain and such that the oscillation amplitude is small outside this subgroup on a long time scale. We use a localization criterion based on the information entropy and verify numerically that such localized excitations exist when the nonlinear dynamics of the subgroup oscillates with a frequency inside the reactive band of the linear chain. We predict a mimium value for the Morse parameter $(\mu>2.25)$ (the only parameter of our normalized model), in agreement with the numerical calculations (an estimate for the biological value is $\mu$ =6.3). For supercritical masses, we use canonical perturbation theory to expand the frequencies of the subgroup and we calculate an energy threshold in agreement with the numerical calculations.
\end{abstract}

DOI: 10.1103/PhysRevE.70.026213

PACS number(s): 05.45.-a, 63.20.Ry, 87.14.Gg, 87.15.-v

\section{INTRODUCTION}

A plethora of chemical processes involving the DNA macromolecule are known [1-3], for example, the existence of denaturation bubbles containing a few broken $\mathrm{H}$ bonds, and the transcription process triggered by the bonding of the biochemical complex to a specific region of the DNA (the socalled TATA box). The oscillator-chain model for DNA [4] was first proposed to study the thermal denaturation of the DNA macromolecule, i.e., the separation of the two strands. The dynamics of this model was first approximated with soliton techniques [5-8]. Our motivation for the present work was to study this model with methods of finite-dimensional dynamical systems, which could later be extended to a realistic model of DNA without translation symmetry. In this work we consider a finite chain of $N$ oscillators with initial condition restricted to a small group of $n \ll N$ of consecutive oscillators. We define a localized motion as one in which the amplitude of oscillation is small outside a group of $n_{\max }$ oscillators for all times, with $n<n_{\max } \ll N$, and we introduce a numerical criterion to quantify localization based on the information entropy. We use the correspondence conjecture (CC) of Flach et al. $[9,10]$ that the nonlinear dynamics of the isolated group of $n$ oscillators must have frequencies inside the reactive band of the linearized chain for localization to be possible. Within this conjecture, we show that there is a minimum value for the Morse parameter (the only parameter of the model) for a localized excitation to be possible. The predicted value $\mu=2.25$ agrees with our numerical calculations. Last, since the linear frequencies of the isolated $n$ system lie in the dispersive band, an immediate consequence of the $\mathrm{CC}$ is that there must be a critical nonzero energy for localization (namely, for at least one of the $n$ frequencies to

\footnotetext{
*Electronic address: deluca@df.ufscar.br
}

exit the dispersive band). For supercritical values of the Morse parameter, we use canonical perturbation theory to evaluate the frequency shifts and we predict a threshold energy for localization in agreement with the numerical calculations.

For the normalizations that follow, the most convenient way to introduce the Peyrard-Bishop (PB) model [4] for a DNA macromolecule is by the Lagrangian

$$
\begin{aligned}
L_{\mathrm{PB}}= & \sum_{i=1}^{N} \frac{m}{2}\left(\dot{u}_{i}^{2}+\dot{v}_{i}^{2}\right)-\frac{k}{2}\left(u_{i+1}-u_{i}\right)^{2}-\frac{k}{2}\left(v_{i+1}-v_{i}\right)^{2} \\
& -D\left[\exp a\left(v_{i}-u_{i}\right)-1\right]^{2},
\end{aligned}
$$

where $u_{i}$ and $v_{j}$ denote the relative displacements of the nucleotidic bases at sites $i$ and $j$ of each DNA strand. The number $N$ denotes the number of sites in each strand of the DNA and can be as large as $N \sim 10^{9}$. For practical reasons we perform our numerical experiments with up to $N=500$. The masses of the bases have a common value $m$, the constant $k$ is the longitudinal elastic constant, and the parameters $D$ and $a$ define the Morse potential describing the transverse $\mathrm{H}$ bonds linking the two chains. The experimental values for these parameters have been discussed in the literature [3,13-15]: the mass of the base pairs is about 300 a.m.u. $=5.010^{-25} \mathrm{~kg}$ and the linear spring constant is $0.04 \mathrm{eV} / \AA^{2}$. The hydrogen bond is modeled by the Morse potential with $a=4.45 \AA^{-1}$, and for $D$ we take an average of the value for the guanine-citosine $(\mathrm{G}-\mathrm{C})$ base pair and the value for the thymine-adenine, A-T base pair, $\bar{D}=0.04 \mathrm{eV}$ [13].

By means of a rotation of coordinates defined by

$$
\begin{aligned}
& x_{i}=\left(u_{i}+v_{i}\right) / \sqrt{2}, \\
& y_{i}=\left(u_{i}-v_{i}\right) / \sqrt{2},
\end{aligned}
$$

the PB Lagrangian (1) is split into the sum $L_{\mathrm{PB}}=L_{X}+L_{Y}$, with 


$$
L_{X}=\sum_{i=1}^{N} m \dot{x}_{i}^{2} / 2-k\left(x_{i+1}-x_{i}\right)^{2} / 2,
$$

depending only on the $x$ coordinates, and with $L_{Y}$ depending only on the $y$ coordinates as

$$
L_{Y}=\sum_{i=1}^{N} \frac{1}{2} m \dot{y}_{i}^{2}-\frac{k}{2}\left(y_{i+1}-y_{i}\right)^{2}-\bar{D}\left[\exp \left(-\sqrt{2} a y_{i}\right)-1\right]^{2} .
$$

The Lagrangian $L_{Y}$ can be normalized by introducing a dimensionless time parameter $\tau \equiv \sqrt{\mathrm{k} / \mathrm{m}} t$ and dimensionless coordinates $\xi_{i} \equiv \sqrt{2} a y_{i}$. The above scalings bring $L_{Y}$ of Eq. (4) to the normal form $L_{Y} \equiv k L / 2 a^{2}$, with

$$
L=\sum_{i=1}^{N} \frac{1}{2} \dot{\xi}_{i}^{2}-\frac{1}{2}\left(\xi_{i+1}-\xi_{i}\right)^{2}-\frac{\mu^{2}}{2}\left[\exp \left(-\xi_{i}\right)-1\right]^{2},
$$

where the overdot denotes the derivative with respect to $\tau$. Our normalization differs from that of [3], and it was chosen such that the quartic approximation to Eq. (4) has the form of the Klein-Gordon oscillator chain studied in $[11,12]$. We henceforth study a chain of $N$ sites with periodic boundary conditions described by the Lagrangian (5), a dynamical system depending on the single parameter $\mu^{2} \equiv 4 \bar{D} a^{2} / k$ (henceforth called the Morse parameter). Using the values in the literature [13-15], we estimate a realistic biological value for $\mu$ to be $\mu=6.3$, and the scaling factors for the units of time and energy to be $0.810^{-12} \mathrm{~s}$ and $2.010^{-3} \mathrm{eV}$, respectively.

For small amplitudes, the normal mode frequency spectrum of Eq. (5) is [12]

$$
\omega(k)=\sqrt{\mu^{2}+4 \sin ^{2}(k / 2)},
$$

where $k=(j \pi / N), j=1, \ldots, N$. The range of normal mode frequencies $\mu \leqslant \omega \leqslant \sqrt{\mu^{2}+4}$ constitutes the dispersive band, while the two relations $0 \leqslant \omega<\mu$ and $\omega>\sqrt{\mu^{2}+4}$ define the lower and upper reactive bands, respectively. One expects that localized motions of the chain with frequency components inside the dispersive band will give rise to quasinormal mode excitations, which are typically delocalized in space. In such a way the localized state loses its energy in the form of radiation and spreads out. In contrast, localized excitations displaying only frequency components inside the two reactive bands are expected to preserve localization for long times.

There is a large body of studies of one-dimensional chains, investigating the energy interchange among the "linearized" system modes [16-23]. For initial energy in a few low-frequency modes, one of us (J.D.L.) has developed theoretical descriptions for energy spreading among modes, valid in various energy ranges, which were compared to numerical results for the Fermi-Pasta-Ulam (FPU) chain [16-18] and for the Klein-Gordon chain [12]. If the energy is initially placed in high-frequency modes, the dynamics is transiently mediated by the formation of unstable nonlinear structures [20-23]. The mode energy is found to distribute itself first into a number of structures, localized in space, each consisting of a few oscillators, which coalesce over time into a single localized structure, a chaotic breather (CB). Over longer times the CB is found to break up, with energy transferred to lower-frequency modes. Recently, there has been another set of studies of the discretized KleinGordon equation, from the perspective of studying the stability of breathers, which are chosen as the initial conditions $[24,25]$. For a more comprehensive discussion of the extensive research on the dynamics of oscillator chains, see, for example, Ref. [12].

The study of soliton solutions of the nonlinear partial differential equations obtained by multiple-scale expansions constitutes at present the main line of study of the nonlinear dynamics of DNA models [5,6,8]. Even though the use of modulation equations and soliton theory does furnish interesting results, we made the choice here to follow a different approach, based on normal form methods for lowdimensional Hamiltonian dynamical systems. The reason for this choice is that, as is well known, multiple-scale expansions are valid only for initial data varying slowly in real space and quasimonochromatic in Fourier space, while here we are interested in the evolution of initial excitations strongly localized in real space (delocalized in Fourier space). Moreover, the method used here displays the further advantage that it could be suitably extended to inhomogeneous chain models describing a realistic DNA molecule.

\section{THE CORRESPONDENCE CONJECTURE REVISITED}

In what follows we reconsider the correspondence conjecture of Refs. $[9,10]$ in the light of canonical perturbation theory. With reference to the system (5), let us initially displace from the equilibrium position $\boldsymbol{\xi}=\mathbf{0}$ only a finite number $n \ll N$ of consecutive particles. For such initial datum, instead of studying the dynamics of the full chain, involving a large number of degrees of freedom, we study the dynamics of the subsystem defined by the Lagrangian (5), with the sum restricted to the sites corresponding to the degrees of freedom initially excited, and with fixed end boundary conditions for the next neighbors. Such an $n$-degree of freedom subsystem is thought of as isolated and having energy $E$. This subsystem can be regarded as a perturbation of $n$ linearly coupled oscillators, whose normal mode frequencies $\omega_{1}, \ldots, \omega_{n}$ are shown to lie inside the dispersive band of the larger $N$ chain. For sufficiently low energies, the dynamics is quasilinear and its frequency spectrum is close to the normal mode frequencies $\omega_{1}, \ldots, \omega_{n}$. According to the $\mathrm{CC}$, if one uses such initial conditions for the larger lattice, the normal modes of the larger chain are excited and the initial excitation will spread out, which is what we observe numerically. With increasing energy, the effect of nonlinearity becomes prominent, and the frequency spectrum is modified. In the absence of resonances of third and fourth order (at least) in the harmonic spectrum of the subsystem, the modes preserve their identity and we can follow their frequency shifts inside the dispersive band. According to the $\mathrm{CC}$, one has localization for the initial data at a given energy when the frequencies of the corresponding motion of the subsystem are outside the dispersive band. Of course this can happen only if 
the energy (i.e., the nonlinearity) is high enough.

The conditions required on the frequency spectrum for multiperiodic oscillations are much more restrictive and the localization properties of such states can be very weak [10]. For this reason, we restrict the analysis to periodic oscillations only, which amounts to looking for periodic orbits of the subsystem whose frequency and harmonics lie outside the dispersive band. This analysis is detailed below.

\section{A. Analysis of the finite subsystems}

For the theoretical analysis we consider localized excitations where the amplitude of oscillation is small for sites outside a group of $1+2 r$ modes, say $|i|>r$ (our $i$ runs in both negative and positive directions and the central particle is $i$ $=0$ ). Under this conjecture the dynamics for sites on the right-hand side of the group $(i>r)$ can be approximated by a linear chain driven by the given oscillation of oscillator $r$ (while the same can be said of the oscillators on the left-hand side, $i<-r)$. The equation of motion for the linearized chain can be derived from the Lagrangian (5) by expanding the exponential

$$
\ddot{\xi}_{i}=\xi_{i+1}+\xi_{i-1}-\left(2+\mu^{2}\right) \xi_{i}, \quad i>r,
$$

where the above linearization holds only for the oscillators outside the subgroup, which are supposed to oscillate with a small amplitude $(i>r)$. The coordinate $\xi_{r}(t)$ of oscillator $r$ entering into Eq. (7) must be given a priori as a known forcing term. To solve Eq. (7) by Fourier transform we define the two-component vector

$$
\chi_{i+1} \equiv\left(\begin{array}{c}
x_{i+1}(\omega) \\
x_{i}(\omega)
\end{array}\right)
$$

It can be shown with the help of Eq. (7) that $\chi_{i+1}$ satisfies the linear matrix iteration law

$$
\chi_{i+1}=\left(\begin{array}{cc}
\left(\omega_{o}^{2}-\omega^{2}\right) & -1 \\
1 & 0
\end{array}\right) \chi_{i}
$$

where $\omega_{o}^{2} \equiv 2+\mu^{2}$. For example, in the case of a monochromatic forcing, $\chi_{r+1}(\omega)$ is nonzero only at a single frequency $\bar{\omega}$, and for the iteration of Eq. (9) to produce a bounded amplitude for sites of large $i$ it is necessary that $\left(\bar{\omega}^{2}-\omega_{o}^{2}\right)^{2}>4$, which is the definition of the reactive band (as opposed to the radiation band defined by $\mu<\omega<\sqrt{\mu^{2}+4}$ ). If the forcing has several large Fourier components, the first large component might be in the lower reactive band $(\omega<\mu)$, while the other important harmonics could be in the upper reactive band $\left(\omega>\sqrt{4+\mu^{2}}\right)$.

The first subsystem we consider here (henceforth called the 1 system) is defined by $r=0$ and consists of the nonlinear oscillation of a single particle of coordinate $x_{o}(t)$ with fixed ends $\left(\xi_{-1}=\xi_{1}=0\right)$. This nonlinear dynamics can be derived from the Lagrangian (5) and it is also described by the Hamiltonian



FIG. 1. Frequency of the nonlinear 1 system divided by $\mu$, $(\omega / \mu)$, plotted as a function of the energy for $\mu=2$ (dashed line), $\mu=2.25$ (solid line), and $\mu=2.5$ (dotted line). The horizontal solid line is $\omega / \mu=1$. Notice that at $\mu=2.25$ the frequency line is only tangent to the critical line. Arbitrary units.

$$
H=\frac{1}{2} p^{2}+\xi_{o}^{2}+\frac{\mu^{2}}{2}\left[\exp \left(-\xi_{o}\right)-1\right]^{2} .
$$

The frequency of oscillation for the periodic motion of the Hamiltonian (10) can be determined by a simple quadrature for any energy, by the formula

$$
\omega=\pi\left[\int_{\xi \min }^{\xi \max } \frac{d \xi_{o}}{\sqrt{2 E-2 \xi_{o}^{2}-\mu^{2}\left[\exp \left(-\xi_{o}\right)-1\right]^{2}}}\right]^{-1} .
$$

In Fig. 1 we plot this frequency as a function of the energy for several values of the parameter $\mu$ to illustrate that it is always inside the radiation band for $\mu<2.25$ at any energy. This is then the minimum value for the $\mu$ parameter where localization is possible, as predicted by the correspondence conjecture for the simple 1 system. It turns out that the biological value is $\mu=6.3>2.25$, in agreement with this theory. Another agreement with this simple theory is discussed in the numerical section, as the numerical searches never found a localized state with $\mu<2.5$.

For supercritical values of $\mu(\mu>2.25)$, the frequency (11) is in the lower reactive band for a sufficient large energy. The frequency of small oscillations (zero energy) is easily obtained by expanding the Hamiltonian (10) to quadratic order, and is $\omega_{o}=\sqrt{2+\mu^{2}}>\mu$. The next correction for small energies can be obtained by expanding the Hamiltonian (10) to fourth order in $\xi_{o}$ as

$$
H_{1}^{(4)}=\frac{1}{2} p^{2}+\frac{1}{2} \omega_{o}^{2} \xi_{o}^{2}-\frac{\mu^{2}}{2} \xi_{o}^{3}+\frac{7 \mu^{2}}{24} \xi_{o}^{4},
$$

where the superscript and subscript on $H$ refer to the order of the expansion and to the 1 system, respectively. Introducing action-angle variables and using standard canonical perturbation theory [26], we find that the normal form of the Hamiltonian (12) up to second order in the action variable $J$ is 


$$
\hat{H}_{1}^{(4)}=\omega_{o} J-g(\mu) J^{2},
$$

with

$$
g(\mu)=\frac{\left(4 \mu^{2}-7\right)}{8\left(\mu^{2}+2\right)^{2}} .
$$

Notice that for supercritical values of $\mu(\mu>2.25)$, the coefficient $g(\mu)$ as defined by Eq. (14) is positive $[g(\mu)>0]$, such that the nonlinear frequency decreases with increasing energy. Defining the nonlinear frequency by $\Omega \equiv \partial H_{1}^{(4)} / \partial J$, the conditions $H_{1}^{(4)}=E_{c}$ and $\Omega=\partial H_{1}^{(4)} / \partial J=\mu$ determine the minimum energy $E_{c}$ to be

$$
E_{c}=\frac{\omega_{o}^{2}-\mu^{2}}{4 g(\mu)}=\frac{4\left(\mu^{2}+2\right)^{2}}{\mu^{2}\left(4 \mu^{2}-7\right)} .
$$

The interpretation of Eq. (15) is as follows. If the isolated nonlinear oscillator of the 1 system has an energy $E>E_{c}$, its frequency is in the reactive band $(\Omega<\mu)$ and we expect that the corresponding type (i) initial condition should produce a localized excitation, according to the $\mathrm{CC}$. This determination of the critical energy is compared to the numerical results in the following section, and it turns out to be short by a factor of 2. The explanation for this is that the subsystem consisting of a single oscillator loses a significant amount of energy to its immediate neighbors, such that one could expect a higher critical energy. It turns out that the value $E_{c} \simeq 1$ predicted by Eq. (15) is precisely a factor of 2 short of the numerical value $E_{c} \simeq 2$ for any value of $\mu$. Our simple theory is then seen to be in only qualitative agreement with the numerical calculations. A better approximation should be given by a subsystem consisting of three particles with fixed ends, which is our next subsystem.
We consider another subsystem (henceforth called the 3 system), consisting of three oscillators along the symmetric motion defined by $\xi_{-1}=\xi_{1}$. The Lagrangian equations of motion derived from Eq. (5) with the condition $\xi_{-1}=\xi_{1}$ correspond to the following two-degrees-of freedom Hamiltonian:

$$
\begin{aligned}
H_{3}= & \frac{1}{2} p_{o}^{2}+\frac{1}{4} p_{1}^{2}+\xi_{1}^{2}+\left(\xi_{o}-\xi_{1}\right)^{2}+\mu^{2}\left[\exp \left(-\xi_{1}\right)-1\right]^{2} \\
& +\frac{\mu^{2}}{2}\left[\exp \left(-\xi_{o}\right)-1\right]^{2} .
\end{aligned}
$$

The two frequencies of the quasiperiodic linear motion at zero energy are

$$
\begin{aligned}
& \omega_{1}=\sqrt{\mu^{2}+2-\sqrt{2}}, \\
& \omega_{2}=\sqrt{\mu^{2}+2+\sqrt{2}},
\end{aligned}
$$

which are inside the dispersive band for any $\mu$.

For small energy subsystems Eq. (16) is a perturbation of two harmonic oscillators with frequencies $\omega_{1}$ and $\omega_{2}$ inside the dispersive band of the whole linearized chain. To compute the leading contribution to the frequency shift of each oscillator, we must evaluate the next frequency correction in powers of the actions. One can check that there is no resonance up to fourth order involving the linear part of Eq. (16), i.e., $\omega_{1} / \omega_{2} \neq 1 / 2,1 / 3$. Using canonical perturbation theory [26] we can remove the cubic term from Eq. (16), average the quartic term, and express the normal form of the Hamiltonian (16) up to second order in the actions as

$$
\hat{H}_{3}^{(4)}\left(J_{1}, J_{2}\right)=\omega_{1} J_{1}+\omega_{2} J_{2}-c_{1} J_{1}^{2}-c_{2} J_{2}^{2}-c_{12} J_{1} J_{2},
$$

where the $J$ 's are the action variables and $c_{1}, c_{2}$, and $c_{12}$ are given by

$$
\begin{gathered}
c_{1}=\frac{3 \mu^{2}\left[12 \mu^{6}+(65-26 \sqrt{2}) \mu^{4}+(73-75 \sqrt{2}) \mu^{2}-(42-35 \sqrt{2})\right]}{64 \omega_{1}^{4} \omega_{2}^{2}\left(4 \omega_{1}^{2}-\omega_{2}^{2}\right)}, \\
c_{12}=\frac{3 \mu^{2}\left[36 \mu^{8}+147 \mu^{6}+4 \mu^{4}-278 \mu^{2}-98\right]}{16 \omega_{1}^{3} \omega_{2}^{3}\left(4 \omega_{1}^{2}-\omega_{2}^{2}\right)\left(4 \omega_{2}^{2}-\omega_{1}^{2}\right)}, \\
c_{2}=\frac{3 \mu^{2}\left[12 \mu^{6}+(65+26 \sqrt{2}) \mu^{4}+(73+75 \sqrt{2}) \mu^{2}-(42+35 \sqrt{2})\right]}{64 \omega_{1}^{2} \omega_{2}^{4}\left(4 \omega_{2}^{2}-\omega_{1}^{2}\right)} .
\end{gathered}
$$

The nonlinearly modified frequencies are given by

$$
\begin{aligned}
& \Omega_{1}=\frac{\partial \hat{H}}{\partial J_{1}}=\omega_{1}-2 c_{1} J_{1}-c_{12} J_{2}, \\
& \Omega_{2}=\frac{\partial \hat{H}}{\partial J_{2}}=\omega_{2}-2 c_{2} J_{2}-c_{12} J_{1} .
\end{aligned}
$$

For supercritical values of $\mu$ the coefficients of Eq. (19) are all positive, such that the frequencies of Eq. (20) are decreasing functions of the energy. The two periodic orbits branching from the linear modes of Eq. (18) are obtained by setting one of the actions of Eq. (18) to zero. For example, by substituting $J_{2}=0$ into Eq. (18) we obtain

$$
\hat{H}_{3}^{(4)}\left(J_{1}, 0\right)=\omega_{1} J_{1}-c_{1} J_{1}^{2},
$$

such that the critical energy predicted by setting $\Omega_{1}=\mu$ is 


$$
E_{c}^{(1)}=\frac{\omega_{1}^{2}-\mu^{2}}{4 c_{1}}=\frac{2-\sqrt{2}}{4 c_{1}} .
$$

For the other periodic orbit we substitute $J_{1}=0$ into Eq. (18), yielding

$$
\hat{H}_{3}^{(4)}\left(0, J_{2}\right)=\omega_{2} J_{2}-c_{2} J_{2}^{2},
$$

and the critical energy predicted by setting $\Omega_{2}=\mu$ is

$$
E_{c}^{(2)}=\frac{\omega_{2}^{2}-\mu^{2}}{4 c_{2}}=\frac{2+\sqrt{2}}{4 c_{2}} .
$$

For values of $\mu$ in the interval $(2.5<\mu<30)$ one sees that the values of $c_{1}$ and $c_{2}$ are close to the limiting values $c_{1}$ $\simeq c_{2} \simeq \frac{3}{16}$, while $c_{12}$ has the limiting value $c_{12} \simeq \frac{3}{4}$. [It is easy to obtain this limit by setting $\omega_{1} \sim \omega_{2} \sim \mu$ and $4 \omega_{1}^{2}-\omega_{2}^{2}$ $\sim 4 \omega_{2}^{2}-\omega_{1}^{2} \sim 3 \mu^{2}$ into the formulas of Eq. (19).] The limiting values for the critical energies are $E_{c}^{(1)}=4(2-\sqrt{2}) / 3 \simeq 0.78$ and $E_{c}^{(2)}=4(2+\sqrt{2}) / 3=4.55$. The critical energy $E_{c}^{(1)}=0.78$, obtained for localized excitations generated by $J_{2}=0$, agrees within $25 \%$ with the numerical calculations of the next section, which determine $E_{c} \simeq 0.6$. For initial conditions in the 3 system, the energy leaking out is compensated by a negative interaction energy of the 3 system with the rest, such that the energy inside the 3 system is actually larger than the total energy (this explains how we have overestimated the critical energy). The reason for this better agreement is still that, by increasing the subsystem size, the interaction energy with the immediate neighbors (whatever its sign) becomes less important. Models with more oscillators in the subgroup should furnish even better approximations, but they are harder to work out analytically and the corresponding type $(n)$ initial conditions are computationally more expensive to investigate.

\section{NUMERICAL RESULTS}

We present numerical results for the DNA oscillator chain, with initial condition in two different types of oscillator groups. All of our numerical integrations were performed with a tenth-order symplectic Runge-Kutta-Nystrom integrator [27]. The high-order integrator can take very large steps, of about 0.6 of the shortest linear period, and still conserve energy with a precision of $10^{-10}$ even after integration times of $10^{10}$.

\section{A. Macroscopic quantities}

The dynamics of the full chain described by the Lagrangian (5) is described by the following Hamiltonian:

$$
H=\sum_{i=-N /}^{N / 2} \frac{1}{2} p_{i}^{2}+\frac{1}{2}\left(\xi_{i+1}-\xi_{i}\right)^{2}+\frac{1}{2} \mu^{2}\left[\exp \left(-\xi_{i}\right)-1\right]^{2} .
$$

In numerical experiments the instantaneous values of the onsite oscillator energies $E_{i}, i=1, \ldots, N$, are usually calculated as

$$
\begin{aligned}
E_{i} \equiv & \frac{1}{2} p_{i}^{2}+\frac{1}{4}\left(\xi_{i+1}-\xi_{i}\right)^{2}+\frac{1}{4}\left(\xi_{i+1}-\xi_{i}\right)^{2} \\
& +\frac{1}{2} \mu^{2}\left[\exp \left(-\xi_{i}\right)-1\right]^{2},
\end{aligned}
$$

where we include $50 \%$ of the interaction with the oscillator at each side, such that the sum of the $E_{i}$ is the constant total energy. Over short times the instantaneous and average values are nearly the same. The information entropy is defined by

$$
S=-\sum_{i=1}^{N} e_{i} \ln e_{i},
$$

where $e_{i}=E_{i} / \Sigma_{i}^{N} E_{i}$ are the normalized instantaneous oscillator energies. In a typical situation where the total energy is distributed among $r<N$ oscillators, $r$ of the $e_{i}$ are of order $1 / r$ and the remaining are negligible, such that Eq. (27) predicts $S \simeq \ln (r)$. This motivates the definition of

$$
N_{\text {osc }} \equiv \exp (S)
$$

as the effective number of oscillators sharing the energy. It is also convenient to define the normalized parameter

$$
n_{\mathrm{osc}} \equiv N_{\mathrm{osc}} / N
$$

The normalized parameter $n_{\text {osc }}$ varies from 0 to 1 , because the entropy of Eq. (27) is always less than $\ln (N)$. The instantaneous value of $n_{\text {osc }}$ does not asymptote to 1 , due to fluctuations. To calculate the effect of fluctuations we introduce a deviation $\delta e_{i}$ from equipartition $e_{i}=\bar{e}+\delta e_{i}$. Substituting this into Eq. (29), expanding the logarithm function as $\ln (1$ $\left.+\delta e_{i} / \bar{e}\right)=\delta e_{i} / \bar{e}-(1 / 2)\left(\delta e_{i} / \bar{e}\right)^{2}$, and performing the summation over $i$ yields

$n_{\mathrm{osc}}=\frac{1}{N} \exp \left\{-N \bar{e} \ln (\bar{e})-N(\delta e)^{2} /(2 \bar{e})\right\}=\exp \left\{-N(\delta \bar{e})^{2} /(2 \bar{e})\right\}$.

Taking $\bar{e}=1 / N$ and making the assumption of normal statistics, that for each normal mode $(\delta \bar{e})^{2}=\bar{e}^{2}$ (which is true only for linearized lattice dynamics), we see that $N$ cancels, giving an asymptotic value $n_{\text {osc }}=\exp (-0.5)=0.61$. This calculation shows that the result does not depend on the number of oscillators if $N$ is large and also shows why the value is different from unity. More accurate calculations have been made, including the nonlinear terms in the oscillator calculation, yielding [23]

$$
n_{\text {osc }}=0.74
$$

at equipartition of energy among the oscillators. These values have been checked numerically, giving good agreement [23].

Numerical experiments show that for a randomly chosen localized initial condition the value of $n_{\text {osc }}$ usually starts to increase and reaches the equipartition value $n_{\mathrm{osc}}=0.61$ in a time of the order of $N$, which is the typical spread time. Our localization criterion is that a state is localized when the value of $n_{\text {osc }}$ is significantly less than the equipartition value 0.61 for more than $50 N$ periods $T_{f}$ of the fastest linear mode 


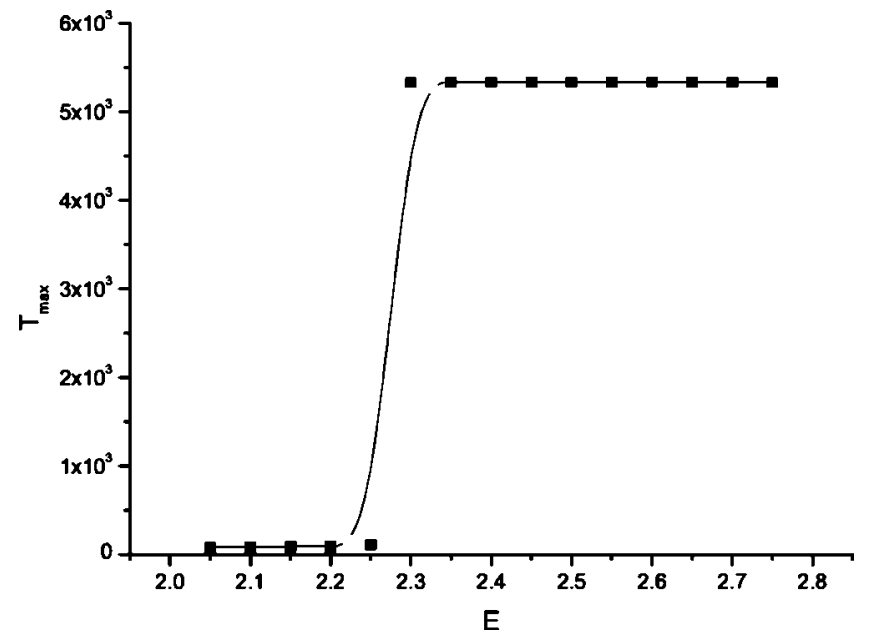

FIG. 2. $T_{\max }$ as a function of the energy for type (i) initial conditions at $N=100$ and $\mu=6.3$. Arbitrary units. The squares represent numerical calculations and the solid line is a spline interpolation.

$\left(T_{f}=2 \pi / \sqrt{\mu^{2}+4} \sim 1\right)$. For the computationally accessible finite values of $N$ (of the order of 100), the smallest value of $n_{\mathrm{osc}}$ is obtained for localization in a single site, $n_{\mathrm{osc}}=1 / \mathrm{N}$, which is an extreme value. Given that $n_{\mathrm{osc}}=0.61$ means equipartition, our practical criterion is $n_{\mathrm{osc}}<\eta_{L} \equiv(20 / N)$ for $t<50 N$. With this criterion we give the state some room to breathe, allowing the energy to spread over $20 \%$ of the oscillators and then to shrink again to a smaller average value. This practical criterion excludes only very odd localized states, that would have sudden delocalization bursts, which was never observed numerically. In the numerical calculations we use a logarithmic scale for the increasing time, in the natural units of the Lagrangian (5). The rapid fluctuations of the instantaneous values are smoothed by the taking the average of the last five instantaneous values of $n_{\text {osc }}$, which are evaluated at a rate of 25 points per decade in time [at every integer value of $25 \ln (t)]$.

Our numerical experiments integrate the dynamics of the Lagrangian (5) for a chain of $N$ oscillators with periodic boundary conditions and we shall use two types of initial conditions, defined as follows. (i) Initial conditions produced by giving a nonzero position and momentum to a single oscillator and a null value for the positions and momenta of all other oscillators (the value of $n_{\mathrm{osc}}$ at $t=0$ is 1 ). (ii) Symmetric initial conditions produced by giving a nonzero value for three consecutive oscillators with the symmetry $x_{-1}=x_{1}$ and $p_{-1}=p_{1}$ (the value of $n_{\text {osc }}$ at $t=0$ is 3 ). For example, we have used $\eta_{L}=0.2$ and we started several (about 50) initial conditions of type (i) with a given energy. For each initial condition we calculate $n_{\mathrm{osc}}$ along the numerical integration and we stop the integration at the first time that $n_{\mathrm{osc}}$ becomes larger than $\eta_{L}=0.2$, defining a delocalization time for that initial condition. The maximum value of the delocalization time $\left(T_{\max }\right)$ among the 50 initial conditions of the same energy is our measure of localization. Typically, for a chain of $N$ $=100$ oscillators, this value is about $T_{\max } \simeq 100=N$ for subcritical energies; then there is a rapid transition where this value climbs to above $T_{\max }=5000$. In practice, it is necessary

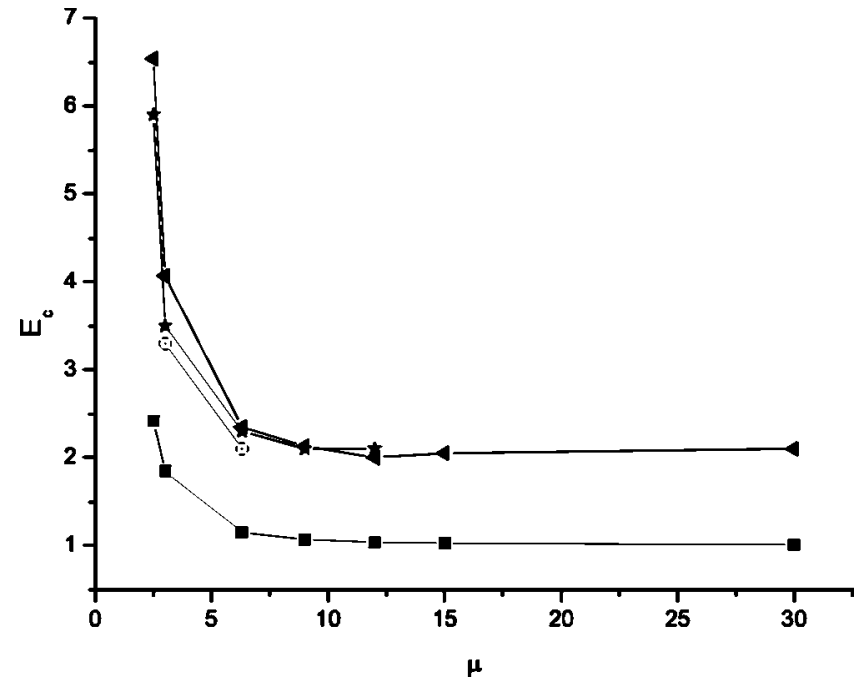

FIG. 3. Numerically calculated critical energy for type (i) initial conditions with $N=100$ (triangles), $N=200$ (stars), and $N=500$ (circles) as a function of the energy. Also plotted is the critical energy predicted by the 1 system (squares). Arbitrary units.

to stop the numerical integration in the supercritical region whenever $T_{\max }$ reaches a maximum value, and we have used $T_{\max }=53 \mathrm{~N}$ as a good computationally accessible large number $(53 N=5300$ if $N=100)$. We experienced with a much higher threshold for $T_{\max }$ of about $1000 \mathrm{~N}$ and obtained virtually the same type of transition, but the numerical experiment became very time consuming. The question of whether this localization time is either infinite, exponentially long, or simply a very large value is not addressed in the present work. We have also varied the threshold value of $n_{\text {osc }}$ among the values $\eta_{L}=0.15, \eta_{L}=0.2$, and $\eta_{L}=25 . N$ and we obtained the same transition line. We used for $N$ the three values $N$ $=100, N=200$, and $N=500$ and obtained virtually the same transition lines for $\mu>3$. A comprehensive statistical analysis has not been performed due to the very long times for

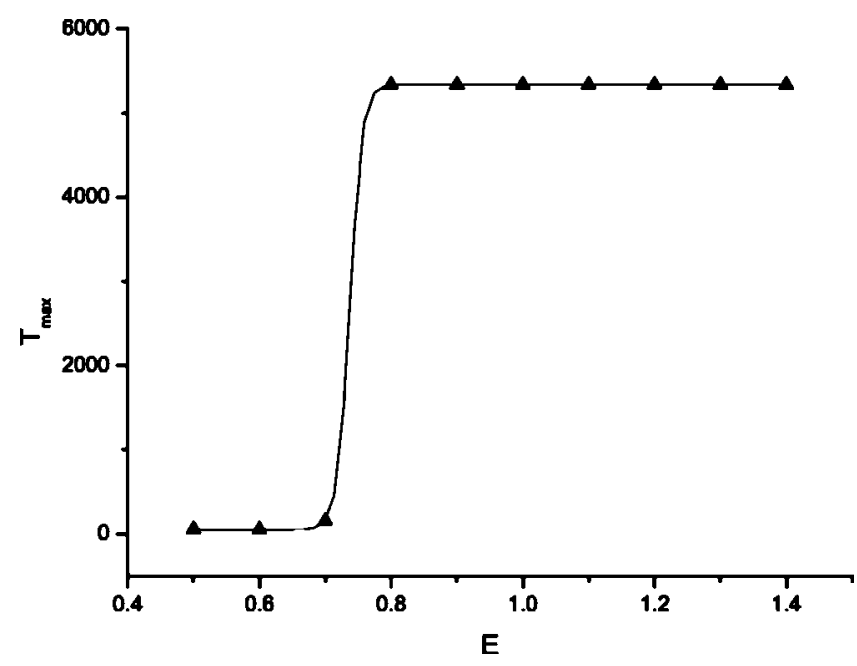

FIG. 4. $T_{\max }$ as a function of the energy for type (ii) initial conditions at $N=100$ and $\mu=3.0$ (triangles). Arbitrary units. The triangles represent the numerical calculations and the solid line is a spline interpolation. 


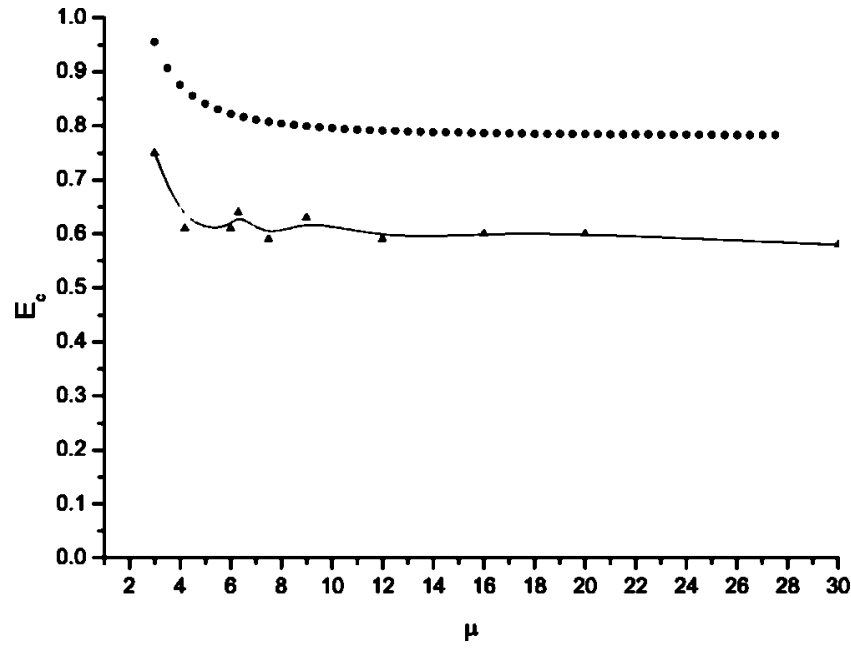

FIG. 5. Numerically calculated critical energy for type (ii) initial conditions with $N=100$ as a function of $\mu$ (triangles) and critical energy predicted by the 3 system $E_{c}^{(1)}$ (circles), as a function of $\mu$. Arbitrary units.

some runs. Spot checks for a few cases indicate that the spread from varying $N$ and $\eta_{L}$ is less than some few percent if $\mu>3$ and $\eta_{L}<(25 / N)$ For the region $2.5<\mu<3$ there can be significant changes in the critical energies determined by the above procedure. This is because close to the critical value $\mu=2.25$ the localization length becomes long, and in a lattice with a small $N$ this localization is confused with equipartition by our criterion. It is interesting to recall that biology chose the safe value of $\mu=6.3$ possibly for the same reason.

In Fig. 2 we plot the value of $T_{\max }<53 \mathrm{~N}$ among 49 initial conditions of type (i) as a function of the energy for $\mu=6.3$. Notice the pronounced jump in $T_{\max }$ which is a signature of localization. We define the critical energy by the inflection point of the $T_{\max }$ curve, which from Fig. 2 is about $E \simeq 2.3$. This same discontinuous behavior of $T_{\max }$ is observed in the numerical calculations for $2.5<\mu<30$, and in Fig. 3 we plot the critical energy determined by the inflection point of $T_{\max }$ and the theoretical predictions for the 1 system, Eq. (15), versus $\mu$. The numerically determined critical energy is about twice that predicted for the simple 1 system by perturbation theory. This effect is due to the fact that for type (i) initial conditions a substantial part of the energy leaks to the immediate neighbors even when there is localization, such that the total energy of the system at localization is significantly larger than the energy of the 1 system.

In Fig. 4 we plot $T_{\max }<53 \mathrm{~N}$ among 49 type (ii) initial conditions, as a function of the energy for $\mu=3.0$ and $N$ $=100$, illustrating the same jump that is our signature of localization. The critical energy predicted by the inflection point of Fig. 4 is $E=0.75$. In Fig. 5 we plot the critical energy determined by the inflection point of $T_{\max }$ and the theoretical predictions of the 3 system versus $\mu$. The theoretical prediction for the 3 system agrees with the numerical results within $25 \%$. The approximation is better than in the case of the 1 system because less energy leaks out of the 3 system. For type (ii) initial conditions there is an interaction term in the total energy that increases the energy inside the 3 system above the total energy, but the predicted energy is now only $25 \%$ wrong.

In Fig. 6 we plot the modulus of the complex Fourier transform of the coordinate of the central oscillator for an initial condition of type (i) of a lattice with $\mu=6.3, N=100$, and a subcritical energy $E=0.1$. Notice that the Fourier transform develops nonzero components inside the conduction band $6.3<\omega<6.61$, as illustrated in the inset to Fig. 6 .

In Fig. 7 we plot the Fourier transform of the coordinate of the central oscillator for a localized initial condition of type (i) in a lattice with $\mu=6.3, N=100$, and a supercritical energy $E=3.0$, which has a primary peak at $\omega=6.0<\mu$ and goes to zero already at $\omega=6.25<\mu$, in accordance with the CC.

Last, in Fig. 8 we plot the surface of section of the 3 system with $\mu=6.3$ at the supercritical energy $E=3$, showing very little stochasticity, to illustrate that localization has nothing to do with stochasticity within the subgroup, as discussed in Ref. [10].



FIG. 6. Fourier transform of an initial condition of type (i) with $E=0.1$ (subcritical) for a lattice with $\mu=6.3$ and $N=100$. Plotted is the modulus $F(\omega)$ of the complex Fourier transform. The inset magnifies the region near $\omega=6.3$ to display that $F(\omega)$ is not zero inside the conduction band. 


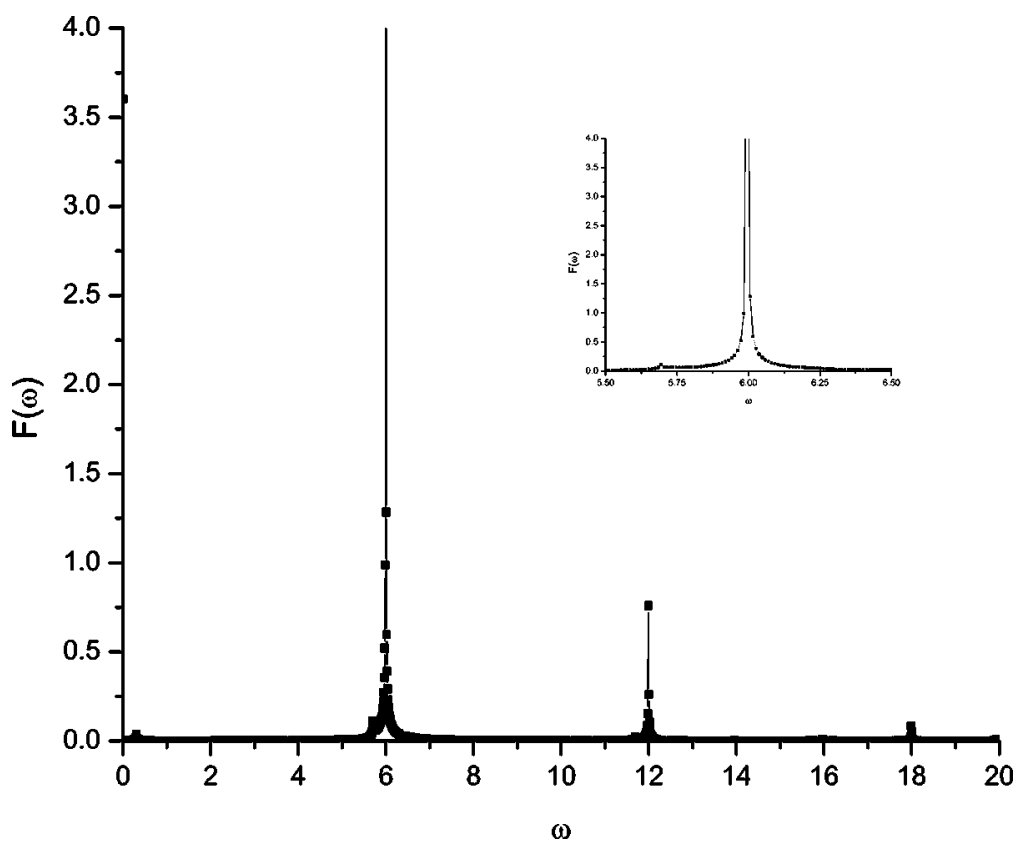

FIG. 7. Fourier transform of an initial condition of type (i) with $E=3.0$ for a lattice with $\mu$ $=6.3$ and $N=100$. Plotted is the modulus $F(\omega)$ of the complex Fourier transform. The inset magnifies the region near $\omega=6.0$ to display the peak of $F(\omega)$ at $\omega=6.0<\mu$. Notice that $F(\omega)$ vanishes above $\omega=6.25<\mu$.

\section{DISCUSSIONS AND CONCLUSION}

At a supercritical energy, by searching among 49 initial conditions of type (i), for example, we have found several initial conditions that stay localized for more than $10^{5}$ natural units. Using a numerical search that varies the initial condition in the neighborhood of an original localized condition [28], in a way that maximizes the localization time, we could easily find other initial conditions that stay localized for a much larger time, of the order of $10^{7}$. These refined initial conditions become restricted to narrow domains, and we believe that the study of time scales for a localized excitation in a chain with a finite $N$ should start from here in future work,

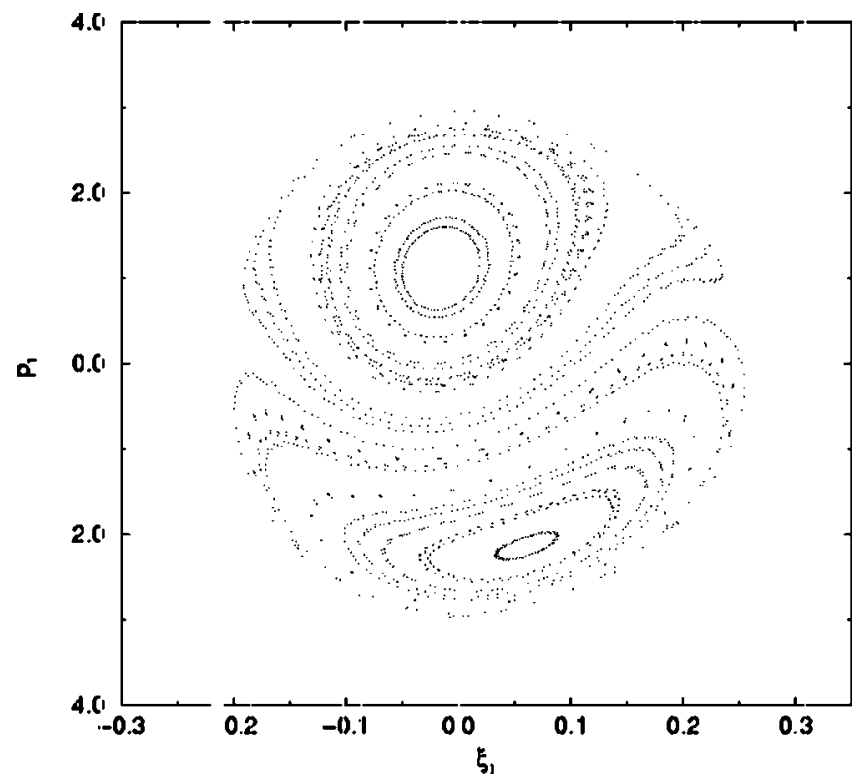

FIG. 8. Surface of section of the symmetric 3 system at $\mu$ $=6.3$ and $E=3.0$, showing little stochasticity. Arbitrary units. for example, to test if one can increase this time arbitrarily.

At a finite $N$, if the energy of a type (ii) initial condition does not localize in the original 3 system, it will leak out to the other $(N / 3) 3$ systems of the chain. A simple condition for these other 3 systems to be "sufficiently linear" is then that the total energy be less than $N E_{c} / 3$ (such that the other 3 systems display a quasilinear motion). This intensive condition $E<N E_{c} / 3$ is important to remember in numerical experiments with a finite lattice. For example, for a chain of $N=100$ oscillators, this means $E<26.4$ and in our numerical experiments we have always stayed well below this energy.

The critical value of $\mu$ for localization $(\mu=2.25)$ is in agreement with the numerical calculations, as we never found localization below $\mu=2.5$. In the region $2.5<\mu<3.0$, the numerical results indicate that the localization length is very large, which requires numerical experiments with large values of $N$. The value $\mu=6.3$ estimated from the biological measurements is far from the critical and in a region where localization length is small, such that we predict a robust localization from the above DNA model. The threshold energy for localization at $\mu=6.3$ is 2.2 units or $4.4 \times 10^{-3} \mathrm{eV}$ $\left(0.17 k_{B} T\right.$ at room temperature). This means that localization is possible at room temperature, as predicted by our model. Last, the localization time found numerically is greater than $10^{5}$ time units or $10^{-7} \mathrm{~s}$, enough for the biochemical mechanisms to operate. Such localization can be related to the bubbles in DNA and it would be an auxiliary mechanism in the transcription process.

\section{ACKNOWLEDGMENTS}

We thank Allan Lichtenberg for discussions on breather research. We acknowledge a Fapesp grant which supported A.P. in Brazil, where this work was completed. A.P. acknowledges GNFM for the payment of travel expenses. J.D.L. and E.D.F. acknowledge partial support from CNPQ. 
[1] W. Saenger, Principles of Nucleotidic Acid Structure (Springer-Verlag, New York, 1984).

[2] C. R. Calladine and H. R. Drew, Understanding DNA: The Molecule and How It Works (Academic, Cambridge, England, 1997).

[3] M. Peyrard, Nonlinearity 17, R1 (2004).

[4] M. Peyrard and A. R. Bishop, Phys. Rev. Lett. 62, 2755 (1989).

[5] M. Techera, L. L. Daemen, and E. W. Prohofsky, Phys. Rev. A 40, 6636 (1989).

[6] G. Gaeta, C. Reiss, M. Peyrard, and T. Dauxois, Nuovo Cimento Soc. Ital. Fis., D 17, 1 (1994).

[7] G. Gaeta, J. Biol. Phys. 24, 81 (1999).

[8] M. Barbi, S. Cocco, M. Peyrard, and S. Ruffo, J. Biol. Phys. 24, 97 (1999).

[9] S. Flach, C. R. Willis, and E. Olbrich, Phys. Rev. E 49, 836 (1994).

[10] S. Flach and C. R. Willis, Phys. Rep. 295, 181 (1998).

[11] M. Pettini and M. Cerruti-Sola, Phys. Rev. A 44, 975 (1991).

[12] J. De Luca and A. Lichtenberg, Phys. Rev. E 66, 026206 (2002).

[13] Z. G. Kudritskya and V. I. Danilov, J. Theor. Biol. 59, 303 (1976).

[14] E. Drigo-Filho and J. R. Ruggiero, Phys. Rev. A 44, 8435 (1991).

[15] S. Zdravkovic and M. V. Staric, Phys. Scr. 64, 612 (2001).
[16] J. De Luca, A. J. Lichtenberg, and M. A. Lieberman, Chaos 5, 283 (1995).

[17] J. De Luca, A. J. Lichtenberg, and S. Ruffo, Phys. Rev. E 51, 2877 (1995).

[18] J. De Luca, A. J. Lichtenberg, and S. Ruffo, Phys. Rev. E 60, 3781 (1999).

[19] C. F. Driscoll and T. M. O 'Neil, Phys. Rev. Lett. 37, 69 (1976); Rocky Mt. J. Math. 8, 211 (1978).

[20] V. M. Burlakov, S. A. Kiselev, and V. I. Rupasov, Phys. Lett. A 147, 130 (1990); V. M. Burlakov and S. Kiselev, Zh. Eksp. Teor. Fiz. 99, 1526 (1991) [Sov. Phys. JETP 72, 854 (1991)].

[21] T. Cretegny, T. Dauxois, S. Ruffo, and A. Torcini, Physica D 121, 109 (1998).

[22] Yu. A. Kosevich and S. Lepri, Phys. Rev. B 61, 299 (2000).

[23] V. V. Mirnov, A. J. Lichtenberg, and H. Guclu, Physica D 157, 251 (2001).

[24] O. Bang and M. Peyrard, Phys. Rev. E 53, 4143 (1996).

[25] J. L. Marin and S. Aubry, Nonlinearity 9, 1501 (1996); Physica D 119, 163 (1998).

[26] G. Bennettin, L. Galgani, and A. Giorgilli, in Advances in Nonlinear Dynamics and Stochastic Processes, Proceedings of the meeting on nonlinear dynamics, edited by R. Livi and A. Politi (World Scientific, Singapore, 1985).

[27] Ch. Tsitouras, Celest. Mech. Dyn. Astron. 74, 223 (1999).

[28] J. De Luca, Phys. Rev. E 62, 2060 (2000). 